\title{
Synthesis, Characterization, and Photoconductivity Studies on Poly(2-chloroaniline) and Poly(2-chloroaniline)/CuO Nanocomposites
}

\author{
Porselvi Linganathan, ${ }^{1}$ Jency Sundararajan, ${ }^{2}$ and Jhancy Mary Samuel ${ }^{1}$ \\ ${ }^{1}$ Department of Chemistry, Auxilium College, Vellore, Tamil Nadu 632006, India \\ ${ }^{2}$ Department of Electrical Engineering, University of Washington, Seattle, WA 98195, USA \\ Correspondence should be addressed to Jhancy Mary Samuel; jhancy2011@gmail.com
}

Received 19 June 2014; Revised 20 August 2014; Accepted 10 September 2014; Published 14 October 2014

Academic Editor: Suying Wei

Copyright (C) 2014 Porselvi Linganathan et al. This is an open access article distributed under the Creative Commons Attribution License, which permits unrestricted use, distribution, and reproduction in any medium, provided the original work is properly cited.

\begin{abstract}
The poly(2-chloroaniline) and poly(2-chloroaniline)/CuO nanocomposites with various weight percentages (5\%, 10\%, 15\%, 20\%, and $25 \%$ ) were synthesized by in situ chemical oxidative polymerization method using ammonium per sulphate (oxidant), $\mathrm{HCl}$ (dopant), and dodecyl benzene sulphonic acid as a surfactant at $0^{\circ} \mathrm{C}$. The formation of polymer and its composites was confirmed by FTIR and UV-Visible spectroscopy. The SEM and X-ray diffraction studies clearly indicate the uniform dispersion of CuO nanoparticles into the polymer matrix. The thermal stability of the polymer and its composites increased with increase in the percentage of $\mathrm{CuO}$ nanoparticles. The polymer and composites exhibit fluorescence property and hence can be used in the light emitting diodes. The current voltage $(I-V)$ curves clearly illustrate the enhanced conductivity on light exposure compared to the dark current. The conductivity of the polymer increased with increase in the percentage of $\mathrm{CuO}$ nanoparticles. Poly(2chloroaniline $/ \mathrm{CuO}$ nanocomposites with $25 \%$ of $\mathrm{CuO}$ show a maximum conductivity of $2.05 \times 10^{-4} \mathrm{Scm}^{-1}$. The composites synthesized behave as organic metals due to their semiconducting nature.
\end{abstract}

\section{Introduction}

Electrically conducting polymers are a class of synthetic metals that combine the chemical and mechanical properties of polymers with the electronic properties of metals and semiconductors [1]. Typical conducting polymers include polyacetylene, polyaniline, polypyrrole, polythiophene, poly(para phenylene), poly(phenylene vinylene), and polyfuran [2]. Ease of synthesis, environmental stability, ease of transformation in cathodic protection, and special electronic, optical, and magnetic properties attract researchers to extensively study polyaniline in the recent past [3]. In spite of various advantages, polyaniline has certain limitations when it comes to its applications as it is neither soluble nor fusible in organic solvents as well as water [4]. In order to overcome such disadvantages, attempts have been made by the use of molecular design, modification of monomer structure, use of functionalized acid dopant, formation of blends/composites, and copolymerization [5].
Organic-inorganic composites have attracted considerable attention as they can combine the advantages of both components and may offer special properties through reinforcing or modifying each other [6]. To improve and extend the functions of the conducting organic materials, inorganic materials such as metals and metal oxides are often incorporated to form multifunctionalized composites for various applications in the fields of electronics, sensors, catalysis, energy, electromagnetic interference shielding, and biomedicine [7]. The inorganic fillers at nanoscale exhibit high surface to volume ratio and thus are expected to modify the electrical, optical, thermal, and dielectric properties of polymer drastically [8].

Polyaniline and its derivatives have attracted considerable attention to the preparation of its composites with metals and metal oxides, such as conducting polyaniline/WO [9], polyaniline/ZnO- $\mathrm{ZrO}_{2}$ [10], polyaniline $/ \mathrm{Fe}_{3} \mathrm{O}_{4}$ [11], polyaniline/ $\mathrm{TiO}_{2}$ [12], polyaniline/NiO [6], 
polyaniline $/ \mathrm{Fe}_{2} \mathrm{O}_{3}$ [13], polyaniline/Se [14], polyaniline $/ \mathrm{Nb}_{2} \mathrm{O}_{5}$ [15], polyaniline/ $\mathrm{Co}_{3} \mathrm{O}_{4}$ [16], polyaniline/ZnO [17], polyaniline/CdS [18], polyaniline/Cu [19], poly(2chloroaniline) $/ \mathrm{SiO}_{2}$ [1], polyaniline-dodecylbenzenesulfonic acid (DBSA)/organophilic clay [20], polyaniline/ $\mathrm{SnO}_{2}[21]$, polyaniline/CuO [22], and poly(o-chloroaniline)/ZnO [23] nanocomposite systems. However to the best of our knowledge there are no reports on the study of poly(2-chloroaniline)/CuO nanocomposites.

In the present investigation, poly(2-chloroaniline) and poly(2-chloroaniline)/CuO nanocomposites with various weight percentages of $\mathrm{CuO}(5 \%, 10 \%, 15 \%, 20 \%$, and $25 \%)$ were prepared by in situ chemical oxidative polymerization technique using hydrochloric acid as dopant, ammonium per sulphate as an oxidant, and dodecyl benzene sulphonic acid as a surfactant (it can act as another dopant as well) at 0$5^{\circ} \mathrm{C}$. This pathway was selected because one expects that the presence of dodecyl benzene sulphonic acid in the polymer composites enhances the solubility, stability, and conductivity in organic solvents and hence the processability. The resulting polymer and polymer composites were characterized by FTIR, UV, SEM, XRD, and fluorescence studies. The thermal properties were studied by TGA and DTA analysis and the electrical conductivities were measured from the $I-V$ graph using two-point probe method.

\section{Experimental}

2.1. Synthesis of Poly(2-chloroaniline) and Poly(2-chloroaniline)/CuO Nanocomposites. 2-Chloroaniline, ammonium per sulphate, and dodecyl benzene sulphonic acid were purchased from LOBA Chemic, Qualigens, and Avra Synthesis Pvt. Ltd, respectively. $\mathrm{CuO}$ nanoparticles were purchased from Sigma Aldrich.

The poly(2-chloroaniline) and poly(2-chloroaniline)/CuO nanocomposites with various weight percentages $(5 \%, 10 \%, 15 \%, 20 \%$, and $25 \%)$ were synthesized by chemical in situ oxidative polymerization method [24]. The equimolar volumes of $0.6 \mathrm{M}$ solution of 2-chloroaniline and hydrochloric acid were prepared in double distilled water, mixed and kept in the freezing mixture. $\mathrm{CuO}$ was added in various weight percentages $(5 \%, 10 \%, 15 \%, 20 \%$, and $25 \%$ ) to the above solution and kept for vigorous stirring $(30 \mathrm{~min}$ ) to keep the $\mathrm{CuO}$ suspended in the solution. The black coloured $\mathrm{CuO}$ nanoparticles were consistently dispersed in the monomer solution. $0.6 \mathrm{M}$ ammonium per sulphate $(12.78 \mathrm{~g}$ in $100 \mathrm{~mL}$ water) and $0.03 \mathrm{M}$ dodecyl benzene sulphonic acid $(1 \mathrm{~mL}$ in $100 \mathrm{~mL}$ of water) were taken in separate beakers. The oxidant and the surfactant were added slowly to the mixture containing 2-chloroaniline and $\mathrm{CuO}$ nanoparticles for more than 30 minutes and the stirring was continued for six hours. The product was placed in the refrigerator overnight. The green coloured product was filtered, washed, and dried. The same procedure was adopted for the synthesis of poly(2-chloroaniline)/CuO nanocomposites of various weight percentages of $\mathrm{CuO}(5 \%$, $10 \%, 15 \%, 20 \%$, and $25 \%$ ).

Adopting the same procedure, poly(2-chloroaniline) was prepared in the absence of $\mathrm{CuO}$. The polymer composites were obtained in higher yields than the polymer. The weight of the poly(2-chloroaniline) obtained is $3.37 \mathrm{~g}$. The weights of the poly(2-chloroaniline)/CuO nanocomposites with various weight percentages $(5 \%, 10 \%, 15 \%, 20 \%$, and $25 \%)$ are $3.49 \mathrm{~g}$, $3.75 \mathrm{~g}, 4.02 \mathrm{~g}, 4.29 \mathrm{~g}$, and $4.51 \mathrm{~g}$, respectively. The percentage yields of poly(2-chloroaniline)/CuO nanocomposites $(5 \%$, $10 \%, 15 \%, 20 \%$, and $25 \%$ ) when compared to the pure polymer are $3.5 \%, 11.2 \%, 19.3 \%, 27.2 \%$, and $33.8 \%$. When the $\mathrm{CuO}$ weight percentage increases gradually the incorporation of $\mathrm{CuO}$ nanoparticles into the polymer matrix also increases as evident from the results. The polymer and polymer composites were soluble in DMSO and partially soluble in most of the organic solvents.

2.2. Characterization. The FT-IR spectra of poly(2-chloroaniline) and poly(2-chloroaniline/CuO) nanocomposites in $\mathrm{KBr}$ were recorded by Thermo Nicolet, Avatar 370 spectrophotometer from $500 \mathrm{~cm}^{-1}$ to $4000 \mathrm{~cm}^{-1}$. The UV-Visible spectra were recorded from $200-800 \mathrm{~nm}$ using Systronics double beam spectrophotometer 2201. XRD analyses of polymer and polymer composites were performed on a Bruker AXS D8 advance X-ray diffractometer using $\mathrm{Cu}-$ $\mathrm{K} \alpha$, wavelength $1.5406 \AA$. The surface morphology of the polymer and polymer composites was studied using a JEOL model JSM-6390LV Scanning Electron Microscope at an accelerating voltage of $15 \mathrm{KV}$. Thermogravimetric analyses were carried out with a Perkin Elmer STA 6000 at the heating rate of $10^{\circ} \mathrm{C} / \mathrm{min}$ from $40^{\circ} \mathrm{C}$ to $750^{\circ} \mathrm{C}$ under an inert gas atmosphere.

A custom built two-probe optoelectrical measurement unit was used to study the photoconductivity properties of poly(2-chloroaniline) and poly(2-chloroaniline)/CuO nanocomposites with various weight percentages. Schematic representation of the measurement system is displayed in Figure 1. The setup consisted of an optical microscope (bright LED illuminator with 6-watt power), probe station (with tungsten probes from Micromanipulator Company), a Keithley source meter, a nanoammeter, and a computer. The probes from the sample are connected to the Keithley source meter, which was controlled by computer using the lab software. Current voltage $(I-V)$ characteristics in dark and light environments were carried out for poly(2-chloroaniline) and poly(2-chloroaniline)/CuO nanocomposites at atmospheric pressure and room temperature. A sweep voltage of $\pm 10 \mathrm{~V}$ was used to perform the measurements.

\section{Results and Discussion}

3.1. FT-IR Spectroscopy. The FTIR spectrum of pure poly(2chloroaniline) is shown in Figure 2(a). The $\mathrm{N}-\mathrm{H}$ stretching frequency of poly (2-chloroaniline) is observed at $3074 \mathrm{~cm}^{-1}$. The asymmetric and symmetric stretching frequencies of $\mathrm{CH}$ present in dodecyl benzene sulphonic acid (DBSA) are observed at $2924 \mathrm{~cm}^{-1}$ and $2856 \mathrm{~cm}^{-1}$. The characteristic peaks due to quinonoid and benzenoid rings of poly $(0-$ chloroaniline) occur at $1581 \mathrm{~cm}^{-1}$ and $1510 \mathrm{~cm}^{-1}$ [25, 26]. The peak at $1303 \mathrm{~cm}^{-1}$ is due to aromatic C-N stretching vibration. The bands at $918 \mathrm{~cm}^{-1}$ and $833 \mathrm{~cm}^{-1}$ are due to 


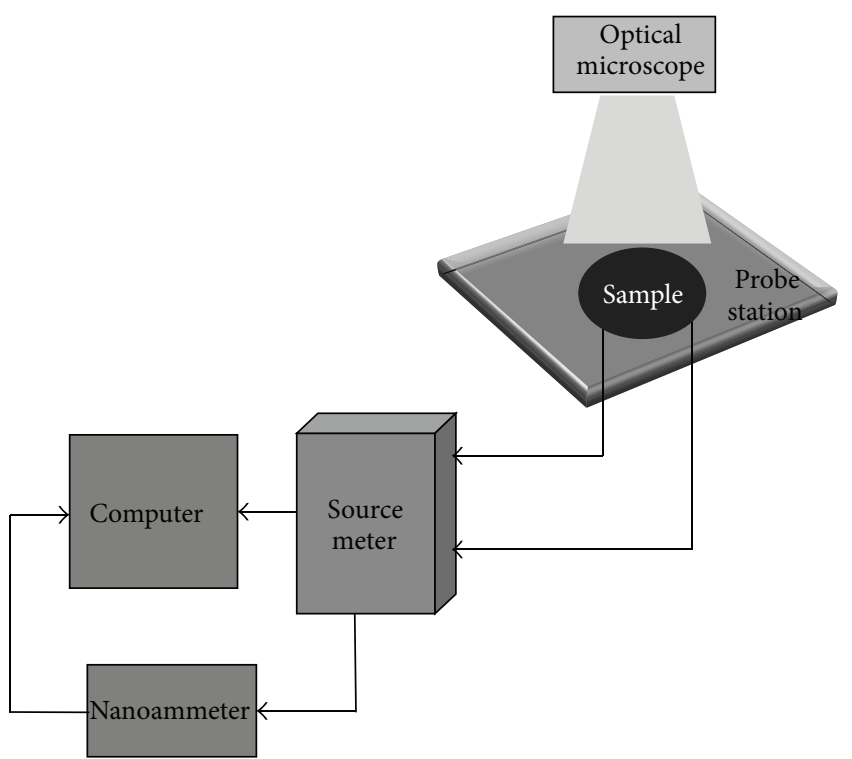

FIGURE 1: Schematic representation of the custom built experimental setup used to carry out electrical measurement.

- $\mathrm{CH}$ out of plane bending vibration of $1,2,4$ tri substituted aromatic rings. The peak at $748 \mathrm{~cm}^{-1}$ corresponds to the chloro group attached to the phenyl ring. All the above peaks confirm the polymerization of 2-chloroaniline.

The FTIR spectra of poly(2-chloroaniline)/CuO with $5 \%$ of $\mathrm{CuO}$ are shown in Figure $2(\mathrm{~b})$. The $\mathrm{N}-\mathrm{H}$ stretching frequency of poly(2-chloroaniline)/CuO is observed at $3066 \mathrm{~cm}^{-1}$. The asymmetric and symmetric stretching frequencies of $-\mathrm{CH}$ present in dodecyl benzene sulphonic acid (DBSA) are observed at $2922 \mathrm{~cm}^{-1}$ and $2854 \mathrm{~cm}^{-1}$. The characteristic peaks due to quinonoid and benzenoid rings of poly (2-chloroaniline) occur at $1579 \mathrm{~cm}^{-1}$ and $1504 \mathrm{~cm}^{-1}$. The peak at $1300 \mathrm{~cm}^{-1}$ is due to aromatic $\mathrm{C}-\mathrm{N}$ stretching vibration. The bands at $898 \mathrm{~cm}^{-1}$ and $827 \mathrm{~cm}^{-1}$ are due to $-\mathrm{CH}$ out of plane bending vibration of 1,2, 4 tri substituted aromatic rings. The peak at $750 \mathrm{~cm}^{-1}$ corresponds to the chloro group attached to the phenyl ring. The characteristic stretching frequencies are shifted towards the lower frequency side in the composite when compared to poly(2-chloroaniline). The above peaks confirm the formation of composite and also suggest a van der Waals kind of interaction between the polymeric chain and $\mathrm{CuO}$ nanoparticles. The same results were found in the polymer composites with $10 \%, 15 \%, 20 \%$, and $25 \%$ of $\mathrm{CuO}$ which confirm the formation of poly(2chloroaniline $/ \mathrm{CuO}$ composites.

3.2. UV-Visible Spectroscopy. The UV-Visible spectra of pure poly(2-chloroaniline) and poly(2-chloroaniline)/CuO nanocomposites with various percentages of $\mathrm{CuO}(5 \%$, $10 \%, 15 \%, 20 \%$, and $25 \%)$ are shown in Figure $3((\mathrm{~A})-$ (F)). The spectra of the poly(2-chloroaniline) and poly(2chloroaniline)/CuO with various weight percentages in DMSO (solvent) show three absorption bands at $\sim 250 \mathrm{~nm}$, $\sim 360 \mathrm{~nm}$, and $\sim 550 \mathrm{~nm}$. The absorption bands $\sim 250 \mathrm{~nm}$ are due to $\pi-\pi^{*}$ transition of the benzenoid rings and $\sim 360 \mathrm{~nm}$ are due to $n-\pi^{*}$ transition of quinonoid rings. The bands around $550 \mathrm{~nm}$ are due to the electron transition between the highest occupied molecular orbital of the benzenoid ring to the lowest unoccupied molecular orbital of the quinonoid ring $[27,28]$. The intensities of the three absorption bands increase with increase in the percentage of $\mathrm{CuO}$. When compared to poly(2-chloroaniline), the poly(2chloroaniline)/CuO nanocomposites are absorbed at higher wavelength. The red shift which is observed in the polymer composites of varying composition is attributed to the presence of $\mathrm{CuO}$ embedded into the polymer chain. This also results in the broadening of the $n-\pi^{*}$ absorption. This type of interaction may be due to the hydrogen bonding between $\mathrm{N}-\mathrm{H}$. . O of the polymer and $\mathrm{CuO}$, respectively.

3.3. X-Ray Diffraction Studies. The XRD patterns of poly(2chloroaniline) and poly(2-chloroaniline)/CuO nanocomposites with various weight percentages $(5 \%, 10 \%, 15 \%, 20 \%$, and $25 \%)$ are compared in Figure $4((\mathrm{~A})-(\mathrm{G}))$. The XRD pattern of $\mathrm{CuO}$ nanoparticles is shown in Figure $4(\mathrm{G})$. Figure $4(\mathrm{~A})$ shows the diffraction peaks (broad) around $2 \theta=7^{\circ}$ and $26^{\circ}$ due to the characteristic peaks of emeraldine salt structure [29]. This also substantiates the absorption in the IR spectrum at $1124 \mathrm{~cm}^{-1}$ which is the electrical conductivity peak due to the emeraldine salt structure. In Figure $4(\mathrm{G})$, the $\mathrm{CuO}$ nanoparticles show two sharp peaks between $2 \theta=30^{\circ}$ and $40^{\circ}$ due to highly crystalline nature of $\mathrm{CuO}$ nanoparticles. In the composites, the high intensity peaks of $\mathrm{CuO}$ were masked by the presence of poly(2-chloroaniline). The peak at $2 \theta=26^{\circ}$ seems to shift to higher wavelength with decreasing intensity as the percentage of $\mathrm{CuO}$ increases. This observation from the XRD data endorses the uniform molecular level dispersion of $\mathrm{CuO}$ nanoparticles into the poly(2-chloroaniline) chain.

3.4. Thermogravimetric Analysis. Thermal degradation patterns of the pristine poly(2-chloroaniline) and poly(2chloroaniline $/ \mathrm{CuO}$ nanocomposites are displayed in Figure 5(a) ((A)-(F)). The derivative curves of poly(2-chloroaniline) and composites are shown in Figure 5(b). The TGA of poly(2-chloroaniline) and poly(2-chloroaniline) $/ \mathrm{CuO}$ nanocomposites undergo four weight loss steps. The first step weight loss around $100^{\circ} \mathrm{C}$ is due to removal of water; the second weight loss around $300^{\circ} \mathrm{C}$ is due to removal of $\mathrm{HCl}$; the third weight loss around $450^{\circ} \mathrm{C}$ is due to removal of dodecyl benzene sulphonic acid dopant; and the exothermic decomposition of poly(2chloroaniline) starts around $500^{\circ} \mathrm{C}$. The decomposition of poly(2-chloroaniline) is continuous up to $700^{\circ} \mathrm{C}$ and even after $700^{\circ} \mathrm{C}$ the complete decomposition has not taken place. The decomposition of the poly(2-chloroaniline) and poly(2-chloroaniline)/CuO nanocomposites leaves some char content. This shows that the presence of chlorine has prevented the complete decomposition. When compared to the poly(2-chloroaniline), the poly(2-chloroaniline)/CuO nanocomposites with various weight percentages show lower weight losses. The poly(2-chloroaniline) has $29.98 \%$ residue and the poly(2-chloroaniline)/CuO nanocomposites with $5 \%, 10 \%, 15 \%, 20 \%$, and $25 \%$ have $35.30 \%, 36.98 \%, 37.00 \%$, $38.38 \%$, and $40.52 \%$ residues, respectively. Though several 


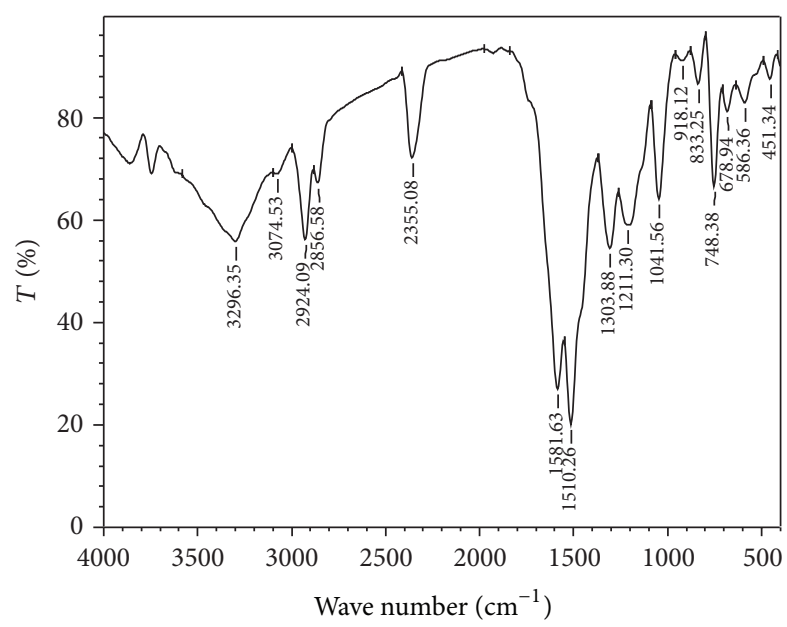

(a)

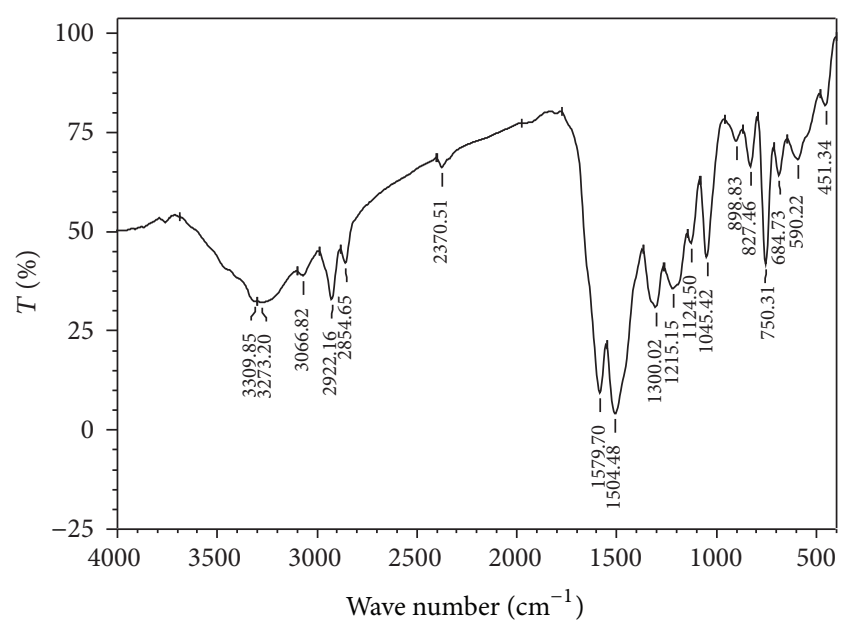

(b)

FIGURE 2: (a) IR spectrum of poly(2-chloroaniline). (b) IR spectrum of poly(2-chloroaniline)/CuO nanocomposite with 5\% CuO nanoparticles.

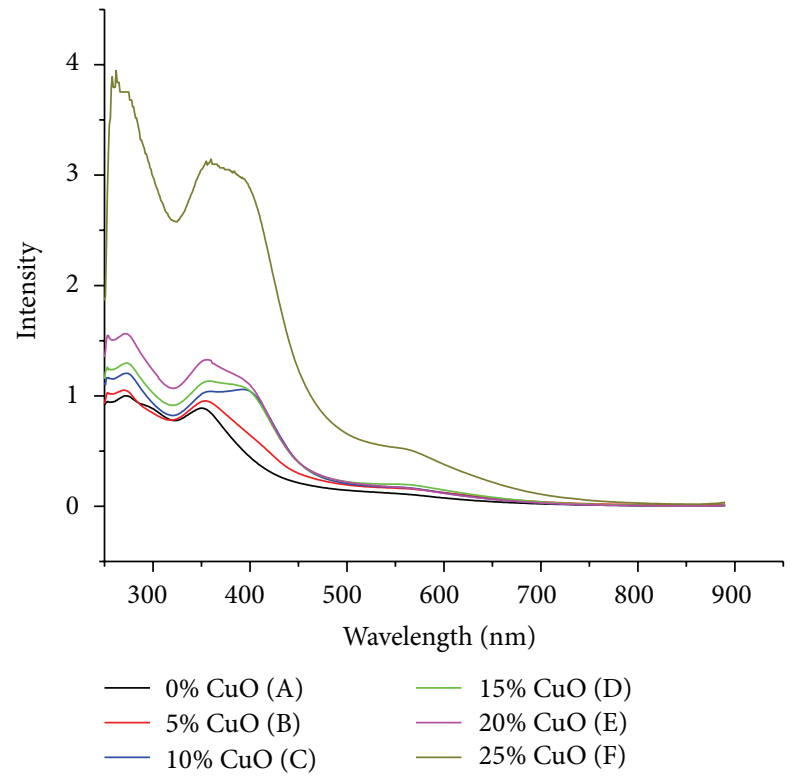

FIGURE 3: UV-Visible spectra of poly(2-chloroaniline) and poly(2chloroaniline)/CuO nanocomposites with various weight percentages of $\mathrm{CuO}$ nanoparticles.

reports demonstrate that the lowered thermal stability of polymer composites with the incorporation of inorganic materials is due to the weakened interfacial interaction between the two components [30,31], in the present work, the thermal stability of the polymer composites improved because of the presence of $\mathrm{CuO}$ nanoparticles. When the temperature is increased the polymer (organic polymer) decomposed continuously but the $\mathrm{CuO}$ present in the polymer matrix does not decompose as the thermal stability of $\mathrm{CuO}$ is high. As the percentage of $\mathrm{CuO}$ increases, the amount of $\mathrm{CuO}$ in the polymer matrix also increases and hence the percentage of residue increases gradually.

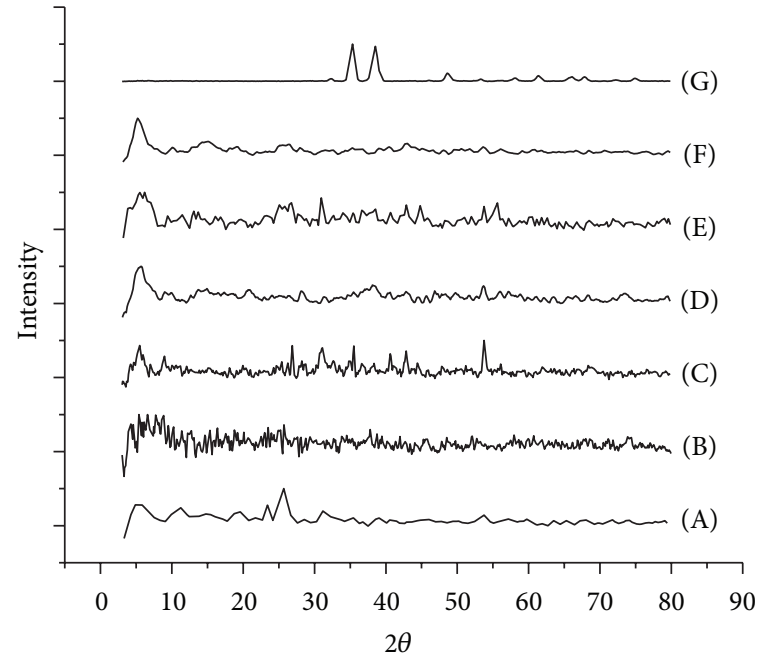

FIGURE 4: XRD patterns of poly(2-chloroaniline) and poly(2chloroaniline) $/ \mathrm{CuO}$ with $5 \%, 10 \%, 15 \%, 20 \%$, and $25 \%$ of $\mathrm{CuO}$ nanoparticles and pure $\mathrm{CuO}$, respectively.

3.5. Surface Morphology. SEM micrographs of the pristine poly(2-chloroaniline) and poly(2-chloroaniline)/CuO nanocomposites containing different amounts of $\mathrm{CuO}$ are displayed in Figures 6(a)-6(f). Pristine poly(2-chloroaniline) (Figure 5(a)(A)) shows an aggregated structure, while poly(2chloroaniline)/CuO nanocomposites exhibit an aggregated grainy morphology. Surface morphology of the poly(2chloroaniline)/CuO nanocomposites with weight percentages $(5 \%$ and $10 \%)$ are shown in Figures $6(\mathrm{~b})$ and $6(\mathrm{c})$. Here the morphology is almost analogous to the pristine poly(2chloroaniline) (Figure 6(a)) because of the lower amount of $\mathrm{CuO}$ nanoparticles, whereas in poly(2-chloroaniline) with weight percentages $20 \%$ and $25 \%$ (Figures $6(\mathrm{e})$ and $6(\mathrm{f})$ ), the visibility of the $\mathrm{CuO}$ particles is very high due to the increase 


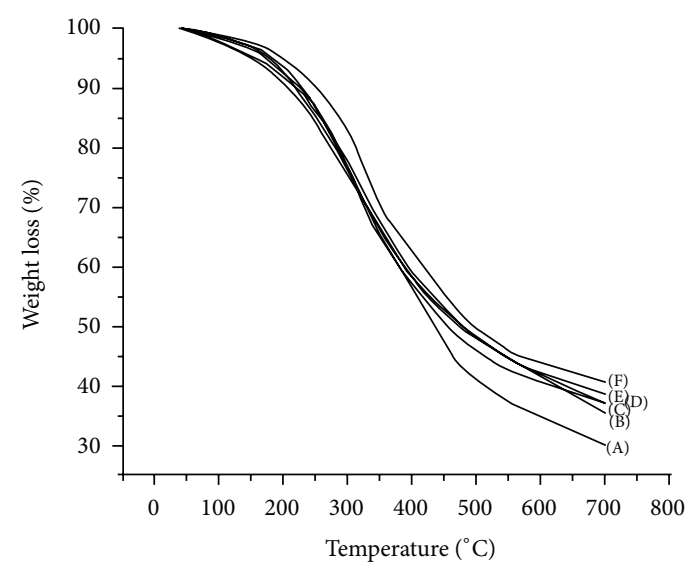

(a)

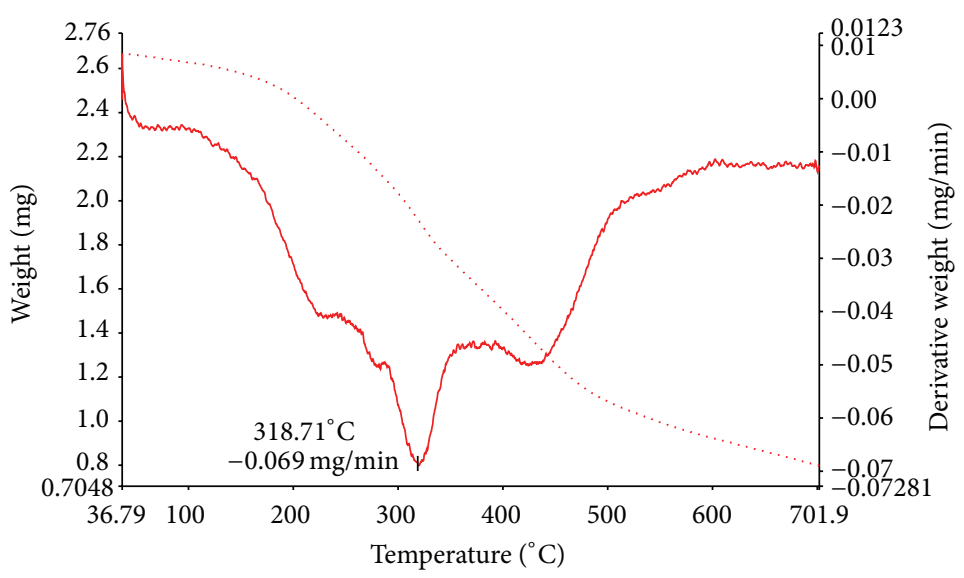

Weight Derivative weight

(b)

FIGURE 5: (a) TGA of poly(2-chloroaniline) and poly(2-chloroaniline)/CuO with $5 \%, 10 \%, 15 \%, 20 \%$, and $25 \%$ of $\mathrm{CuO}$ and pure $\mathrm{CuO}$ nanoparticles, respectively. (b) TGA derivative curve of poly(2-chloroaniline).

in the amount of $\mathrm{CuO}$ nanoparticles in the polymer. But in case of poly(2-chloroaniline)/CuO (15\%) nanocomposites (Figure 6(d)), the perceptibility of the embedded $\mathrm{CuO}$ nanoparticles into the polymer chain is phenomenal. The SEM pictures obviously reveal that the $\mathrm{CuO}$ nanoparticles are embedded in the polymer matrix. The compactness of the polymer nanocomposites is also increased with increase in the $\mathrm{CuO}$ weight percentage. Surface morphology is also an evidence for the uniform dispersion of $\mathrm{CuO}$ nanoparticles into the poly(2-chloroaniline) chain.

3.6. Fluorescence Studies. The fluorescence spectra of poly(2chloroaniline) and poly(2-chloroaniline)/CuO with various weight percentages in dimethyl sulfoxide are shown in Figure 7. The fluorescence of a conjugated polymer can be described in terms of semiconductor band theory. Upon photoexcitation of a conjugated polymer, the electrons from the valence band are excited to the conduction band and then migrated along the polymer backbone. The excited electron and the oppositely charged hole attract one another. When the excited electron combines with the hole a photon is emitted and it is known as fluorescence. The excitation wavelength of polymer and composites is around $350 \mathrm{~nm}$. The poly(2-chloroaniline)/CuO nanocomposites with various weight percentages show emissions around $360 \mathrm{~nm}$, $450 \mathrm{~nm}$, and $730 \mathrm{~nm}$ but the poly(2-chloroaniline) shows emission around $400 \mathrm{~nm}, 450 \mathrm{~nm}$, and $730 \mathrm{~nm}$. The fluorescence spectra clearly illustrate that the poly(2-chloroaniline) and poly(2-chloroaniline)/CuO nanocomposites with various weight percentages have fluorescence properties and can find applications in the manufacture of light emitting diodes.

3.7. Conductivity Studies. The poly(2-chloroaniline) and poly(2-chloroaniline)/CuO nanocomposites with various weight percentages were finely powdered and pressed into pellets using IR Sample Press using Model AVAPAR-330 Thermonicolate applying a maximum pressure of 15 tons $/ \mathrm{cm}^{2}$ to form circular pellets of $1 \mathrm{~cm}$ diameter each. The thicknesses of the pellets were measured using screw gauge. The pellets were used to perform conductivity studies by applying a bias voltage and monitoring the variations in current. Typical $I-V$ characteristic graph of poly(2-chloroaniline) under dark and light (microscopic light) conditions is displayed in Figure 8. The conductivity studies on the sample revealed an enhanced electrical conductivity upon light exposure compared to the dark current. Precisely, an order of magnitude variation in electrical conductivity was observed. The observed enhancement in the current when exposed to the light is mainly due to the photogeneration of charge carriers present in the poly(2chloroaniline) chain. The curves show that in both cases the current increases continuously with the applied voltage. The fluctuations in the current are due to the coarse surface of the poly(2-chloroaniline). The same observations were found in the poly(2-chloroaniline)/CuO nanocomposites with various weight percentages.

The resistance of the poly(2-chloroaniline) and poly(2chloroaniline)/CuO nanocomposites with various weight percentages was determined from the $I-V$ characteristics at a constant voltage $(5 \mathrm{~V})$. Resistances were converted into conductivity using the thickness of the pellet. The resistance and conductivity of poly(2-chloroaniline) and poly(2chloroaniline)/CuO nanocomposites with various weight percentages at constant voltage $(5 \mathrm{~V})$ in the presence and absence of light were tabulated in Tables 1 and 2, respectively.

Figure 9 shows the conductivity data for poly(2-chloroaniline) and poly(2-chloroaniline)/CuO nanocomposites with various weight percentages $(5 \%, 10 \%, 15 \%, 20 \%$, and $25 \%$ ) in the presence and absence of light. The conductivity of the poly(2-chloroaniline)/CuO nanocomposites with various weight percentages shows higher conductivity than the poly(2-chloroaniline). When the percentage of $\mathrm{CuO}$ is increased the conductivity of the polymer is also increased. An order of magnitude increase in conductivity was observed 


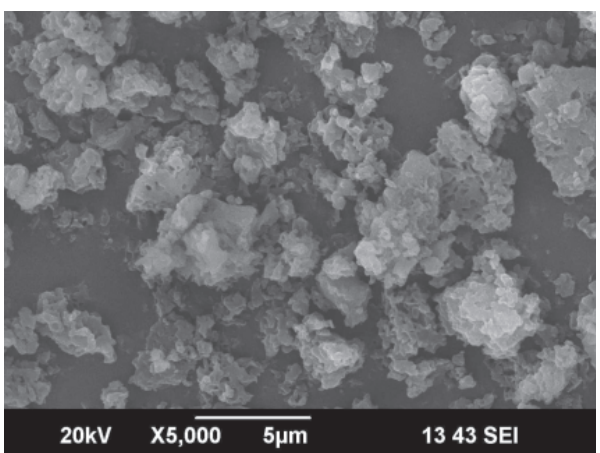

(a)

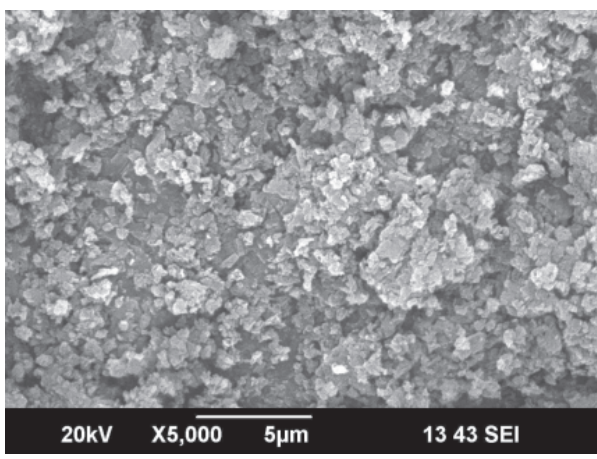

(c)

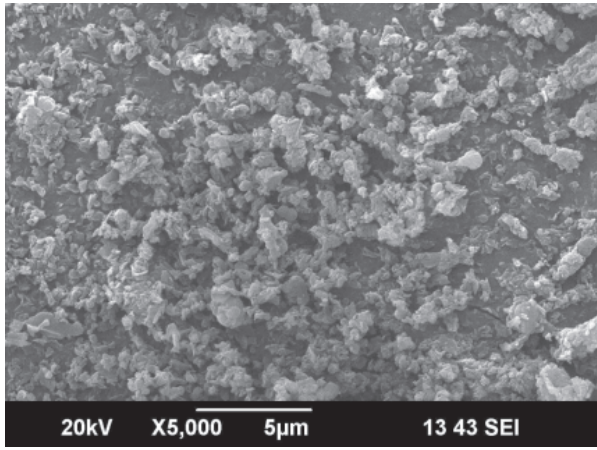

(e)

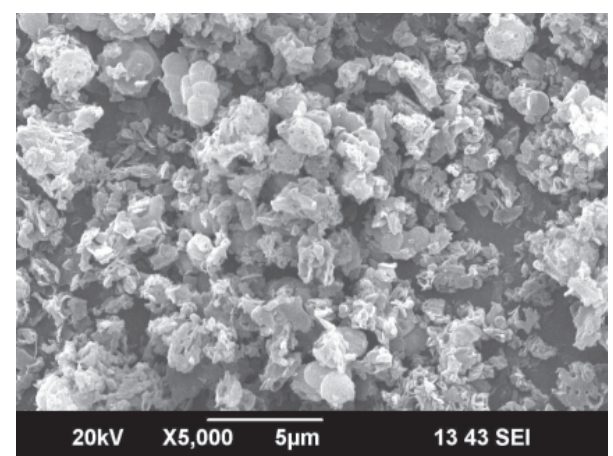

(b)

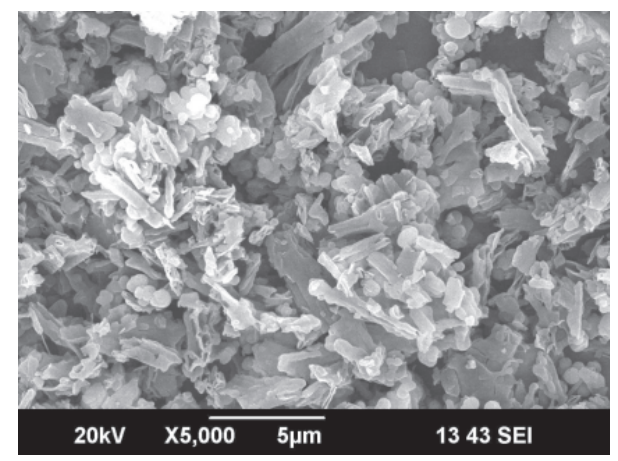

(d)

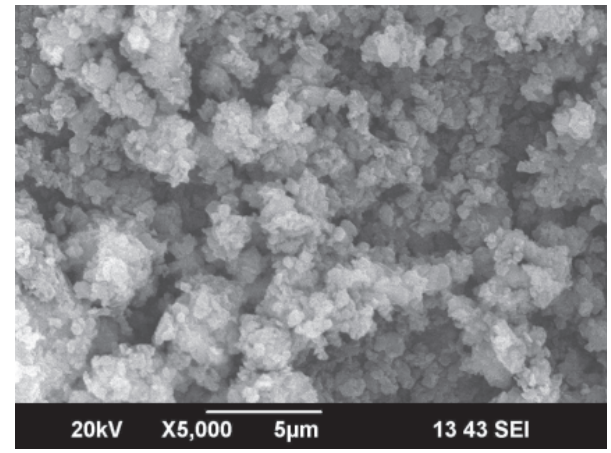

(f)

Figure 6: Surface morphology of poly(2-chloroaniline) and poly(2-chloroaniline)/CuO with 5\%, 10\%, 15\%, 20\%, and 25\% of $\mathrm{CuO}$ nanoparticles, respectively.

in the poly(2-chloroaniline)/CuO nanocomposite (with $25 \%$ of $\mathrm{CuO})$ compared to the poly(2-chloroaniline).

When compared to the conductivity of poly(2-chloroaniline) in the presence and absence of light, high conductivity was detected in the presence of light. The observed enhancement in the current when exposed to the light is mainly due to the photogeneration of charge carriers present in the poly(2chloroaniline) chain. The enhancement of conductivity was also observed in poly(2-chloroaniline)/CuO nanocomposites with various weight percentages inthe presence of light.

\section{Conclusion}

Poly(2-chloroaniline) and poly(2-chloroaniline)/CuO nanocomposites with various weight percentages of $\mathrm{CuO}(5 \%, 10 \%$, $15 \%, 20 \%$, and $25 \%$ ) were successfully synthesized by in situ chemical oxidative polymerization method using ammonium per sulphate (oxidant), $\mathrm{HCl}$ (dopant), and dodecyl benzene sulphonic acid as the surfactant at $0^{\circ} \mathrm{C}$. FTIR spectra of poly(2-chloroaniline) and poly(2-chloroaniline)/CuO nanocomposites with various weight percentages of $\mathrm{CuO}$ nanoparticles show the characteristics peaks with shifts in the wavelength in the case of composites confirming that there is interaction between the poly(2-chloroaniline) and the $\mathrm{CuO}$ nanoparticles. With increasing the percentage of the $\mathrm{CuO}$ the broadening of the peak was also observed in the UV spectrum, indicating that the absorption bands of poly(2-chloroaniline) were influenced by $\mathrm{CuO}$ nanoparticles present in the polymer chain. Poly(2-chloroaniline)/CuO nanocomposites show higher thermal stability than poly(2chloroaniline) and the thermal stability increased with increase in the $\mathrm{CuO}$ percentage. The decreased crystallinity 
TABLE 1: The conductivity of poly(2-chloroaniline) and poly(2-chloroaniline)/CuO nanocomposites in the presence of light.

\begin{tabular}{|c|c|c|c|c|c|}
\hline $\begin{array}{l}\text { Percentage of } \mathrm{CuO} \\
(\%)\end{array}$ & $\begin{array}{c}\text { Current } \\
(\text { at } V=5 \mathrm{~V})\end{array}$ & $\begin{array}{c}\text { Sheet resistance } \\
\left(R_{s}\right)\end{array}$ & $\begin{array}{c}\text { Thickness }(t) \\
(\mathrm{cm})\end{array}$ & Resistivity $(\rho)=R_{s} \times t$ & $\begin{array}{c}\text { Conductivity }=(1 / \rho) \\
\left(\mathrm{S} \mathrm{cm}^{-1}\right)\end{array}$ \\
\hline 0 & $3.10 E-06$ & $1.61 E+06$ & 0.038 & $6.13 E+04$ & $1.63 E-05$ \\
\hline 5 & $8.58 E-06$ & $5.83 E+05$ & 0.066 & $3.84 E+04$ & $2.60 E-05$ \\
\hline 10 & $1.12 E-05$ & $4.46 E+05$ & 0.053 & $2.36 E+04$ & $4.23 E-05$ \\
\hline 15 & $2.10 E-05$ & $2.38 E+05$ & 0.06 & $1.43 E+04$ & $6.99 E-05$ \\
\hline 20 & $2.84 E-05$ & $1.76 E+05$ & 0.075 & $1.32 E+04$ & $7.57 E-05$ \\
\hline 25 & $3.16 E-05$ & $1.58 E+05$ & 0.047 & $7.44 E+03$ & $1.34 E-04$ \\
\hline
\end{tabular}

TABLE 2: The conductivity of poly(2-chloroaniline) and poly(2-chloroaniline)/CuO nanocomposites in the absence of light.

\begin{tabular}{lccccc}
\hline $\begin{array}{l}\text { Percentage of } \mathrm{CuO} \\
(\%)\end{array}$ & $\begin{array}{c}\text { Current } \\
(\text { at } V=5 \mathrm{~V})\end{array}$ & $\begin{array}{c}\text { Sheet resistance } \\
\left(R_{s}\right)\end{array}$ & $\begin{array}{c}\text { Thickness }(t) \\
(\mathrm{cm})\end{array}$ & $\begin{array}{c}\text { Resistivity }(\rho)=R_{s} \times t \\
\begin{array}{c}\text { Conductivity }=(1 / \rho) \\
(\mathrm{S} \mathrm{cm})\end{array}\end{array}$ \\
\hline 0 & $4.05 E-06$ & $1.23 E+06$ & 0.038 & $4.69 E+04$ & $2.13 E-05$ \\
5 & $1.19 E-05$ & $4.19 E+05$ & 0.066 & $2.77 E+04$ & $3.61 E-05$ \\
10 & $1.90 E-05$ & $2.64 E+05$ & 0.053 & $1.40 E+04$ & $7.15 E-05$ \\
15 & $2.29 E-05$ & $2.18 E+05$ & 0.06 & $1.31 E+04$ & $7.63 E-05$ \\
20 & $3.01 E-05$ & $1.66 E+05$ & 0.075 & $1.25 E+04$ & $8.03 E-05$ \\
25 & $4.82 E-05$ & $1.04 E+05$ & 0.047 & $4.88 E+03$ & $2.05 E-04$ \\
\hline
\end{tabular}

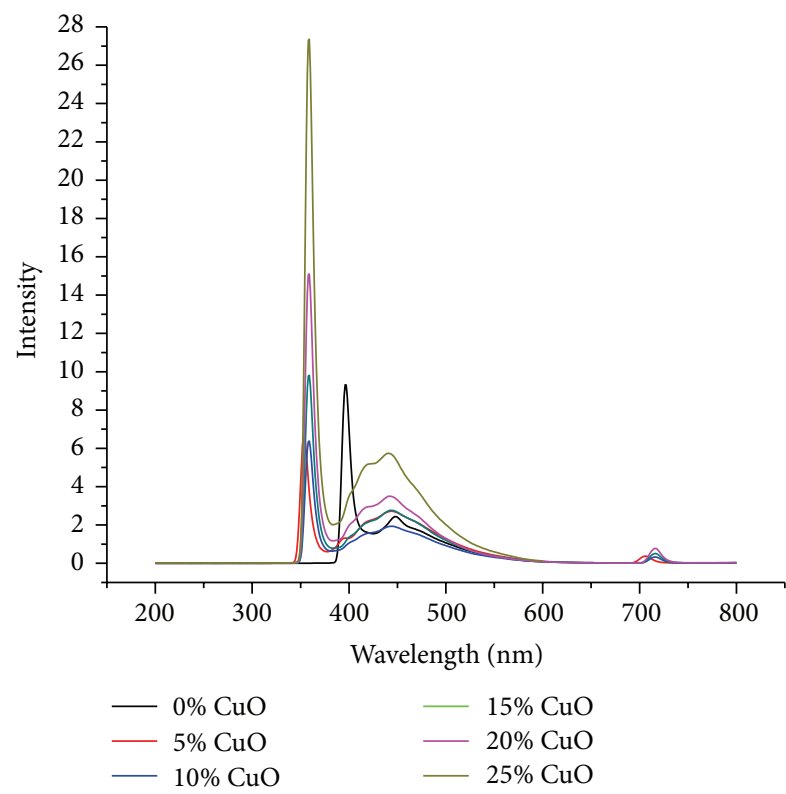

FIGURE 7: Fluorescence spectra of poly(2-chloroaniline) and poly(2chloroaniline)/CuO nanocomposites with various weight percentages of $\mathrm{CuO}$ nanoparticles.

of $\mathrm{CuO}$ nanoparticles in the polymer composites indicates the uniform dispersion of $\mathrm{CuO}$ into the poly(2-chloroaniline) chain. The SEM pictures clearly reveal that $\mathrm{CuO}$ nanoparticles are embedded in the polymer matrix. The compactness of the composites increased with increase of the $\mathrm{CuO}$ percentage. The fluorescence intensity increased with increase in

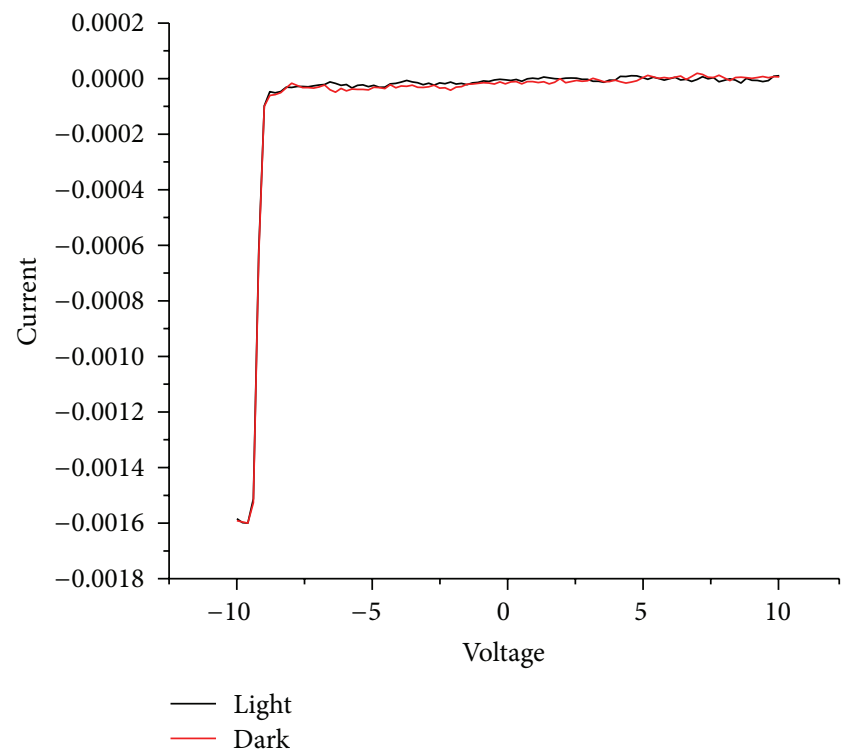

FIGURE 8: Current voltage ( $I-V)$ curves of poly(2-chloroaniline) in the light and dark conditions.

the $\mathrm{CuO}$ nanocomposites. From $I-V$ curves the conductivity of the poly(2-chloroaniline) and poly(2-chloroaniline)/CuO nanocomposites was found in the light and dark condition. The enhanced conductivity was observed in the presence of light due to the excitation of charge carriers in the presence of light. Poly(2-chloroaniline)/CuO nanocomposites with $25 \%$ of $\mathrm{CuO}$ show a maximum conductivity of $2.05 \times 10^{-4} \mathrm{~S} \mathrm{~cm}^{-1}$. 


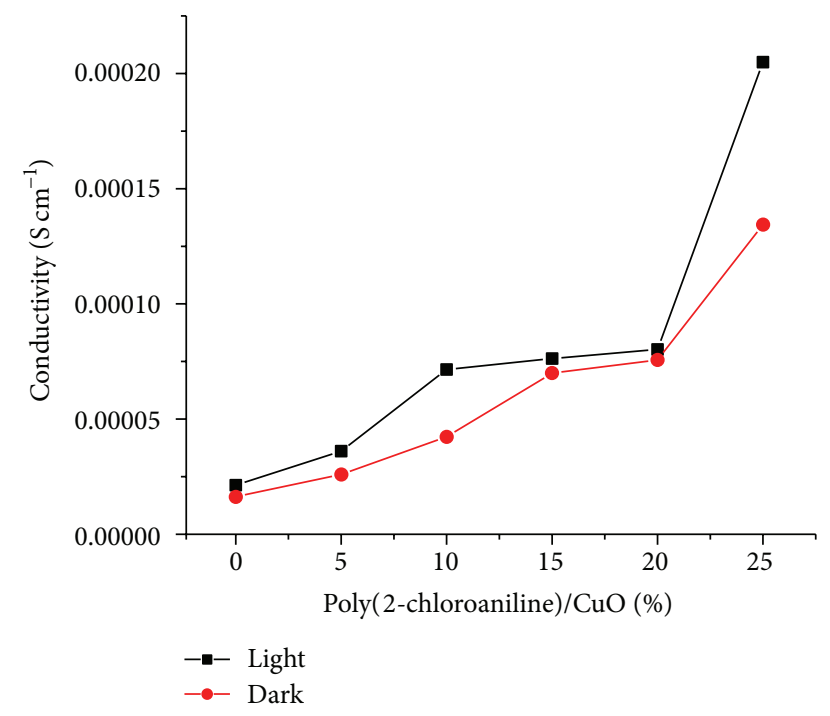

Figure 9: Conductivity of poly(2-chloroaniline) and poly(2chloroaniline) $/ \mathrm{CuO}$ with $5 \%, 10 \%, 15 \%, 20 \%$, and $25 \%$ of $\mathrm{CuO}$ nanoparticles.

\section{Conflict of Interests}

The authors declare that there is no conflict of interests regarding the publication of this paper.

\section{References}

[1] A. Gök and S. Șen, "Preparation and characterization of poly(2chloroaniline) $/ \mathrm{SiO}_{2}$ nanocomposite via oxidative polymerization: comparative UV-vis studies into different solvents of poly(2-chloroaniline) and poly(2-chloroaniline)/ $\mathrm{SiO}_{2}$," Journal of Applied Polymer Science, vol. 102, no. 1, pp. 935-943, 2006.

[2] X. Lu, W. Zhang, C. Wang, T. Wen, and Y. Wei, "Onedimensional conducting polymer nanocomposites: synthesis, properties and applications," Progress in Polymer Science, vol. 36, pp. 671-712, 2011.

[3] A. Liu, L. H. Bac, J.-C. Kim, and L. Liu, "Preparation and characterization of polyaniline-copper composites by electrical explosion of wire," Journal of Nanoscience and Nanotechnology, vol. 12, no. 7, pp. 6031-6035, 2012.

[4] M. Yari and S. Sedaghat, "In-situ synthesis and characterization of conducting metal-polyaniline nanocomposites," Journal of Physical \& Theoretical Chemistry, vol. 5, no. 4, pp. 189-193, 2009.

[5] U. S. Waware and S. S. Umare, "Chemical synthesis, spectral characterization and electrical properties of poly(aniline-co-mchloroaniline)," Reactive and Functional Polymers, vol. 65, no. 3, pp. 343-350, 2005.

[6] B. I. Nandapure, S. B. Kondawar, M. Y. Salunkhe, and A. I. Nandapure, "Magnetic and transport properties of conducting polyaniline/nickel oxide nanocomposites," Advanced Materials Letters, vol. 4, no. 2, pp. 134-140, 2013.

[7] B. I. Nandapure, S. B. Kondawar, and A. I. Nandapure, in Proceedings of International Conference on Benchmarks in Engineering Science and Technoly (ICBEST '12), 2012.

[8] S. L. Patil, M. A. Chougule, S. G. Pawar, S. Sen, and V. B. Patil, "Effect of camphor sulfonic acid doping on structural, morphological, optical and electrical transport properties on
polyaniline-ZnO nanocomposites," Soft Nanoscience Letter, vol. 2, pp. 46-53, 2012.

[9] J. Zhu, S. Wei, L. Zhang et al., "Polyaniline-tungsten oxide metacomposites with tunable electronic properties," Journal of Materials Chemistry, vol. 21, no. 2, pp. 342-348, 2011.

[10] S. Sultana, M. Z. Khan, K. Umar, and M. Muneer, "Electrical, thermal, photocatalytic and antibacterial studies of metallic oxide nanocomposite doped polyaniline," Journal of Materials Science and Technology, vol. 29, no. 9, pp. 795-800, 2013.

[11] A. C. V. de Araújo, R. J. de Oliveira, S. Alves Júnior et al., "Synthesis, characterization and magnetic properties of polyaniline-magnetite nanocomposites," Synthetic Metals, vol. 160, no. 7-8, pp. 685-690, 2010.

[12] M. R. Nabid, M. Golbabaee, A. B. Moghaddam, R. Dinarvand, and R. Sedghi, "Polyaniline/ $/ \mathrm{TiO}_{2}$ nanocomposite: enzymatic synthesis and electrochemical properties," International Journal of Electrochemical Science, vol. 3, no. 10, pp. 1117-1126, 2008.

[13] N. N. Mallikarjuna, S. K. Manohar, P. V. Kulkarni, A. Venkataraman, and T. M. Aminabhavi, "Novel high dielectric constant nanocomposites of polyaniline dispersed with $\gamma-\mathrm{Fe}_{2} \mathrm{O}_{3}$ nanoparticles," Journal of Applied Polymer Science, vol. 97, no. 5, pp. 1868-1874, 2005.

[14] E. Ozkazanc, S. Zor, and H. Ozkazanc, "Synthesis, characterization, and AC conductivity of polyaniline/selenium composites," Journal of Macromolecular Science B: Physics, vol. 51, no. 11, pp. 2122-2132, 2012.

[15] Y. T. Ravikiran, M. T. Lagare, M. Sairam et al., "Synthesis, characterization and low frequency AC conduction of polyaniline/niobium pentoxide composites," Synthetic Metals, vol. 156, no. 16-17, pp. 1139-1147, 2006.

[16] M. D. Bedre, R. Deshpande, B. Salimath, and V. Abbaraju, "Preparation and characterization of polyaniline- $\mathrm{Co}_{3} \mathrm{O}_{4}$ nanocomposites via interfacial polymerization," The American Journal of Materials Science, vol. 2, no. 3, pp. 39-43, 2012.

[17] F. Ahmed, S. Kumar, N. Arshi et al., "Preparation and characterizations of polyaniline (PANI)/ZnO nanocomposites film using solution casting method," Thin Solid Films, vol. 519, no. 23, pp. 8375-8378, 2011.

[18] P. K. Khanna, S. P. Lonkar, V. V. V. S. Subbarao, and K.-W. Jun, "Polyaniline-CdS nanocomposite from organometallic cadmium precursor," Materials Chemistry and Physics, vol. 87, no. 1, pp. 49-52, 2004.

[19] S. Sharma, C. Nirkhe, S. Pethkar, and A. A. Athawale, "Chloroform vapour sensor based on copper/polyaniline nanocomposite," Sensors and Actuators, B: Chemical, vol. 85, no. 1-2, pp. 131-136, 2002.

[20] W. Jia, E. Segal, D. Kornemandel, Y. Lamhot, M. Narkis, and A. Siegmann, "Polyaniline-DBSA/organophilic clay nanocomposites: synthesis and characterization," Synthetic Metals, vol. 128, no. 1, pp. 115-120, 2002.

[21] Z. Hu, Y. Xie, Y. Wang, L. Mo, Y. Yang, and Z. Zhang, "Polyaniline $/ \mathrm{SnO}_{2}$ nanocomposite for supercapacitor applications," Materials Chemistry and Physics, vol. 114, no. 2-3, pp. 990-995, 2009.

[22] D. M. Jundale, S. T. Navale, G. D. Khuspe, D. S. Dalavi, P. S. Patil, and V. B. Patil, "Polyaniline-CuO hybrid nanocomposites: synthesis, structural, morphological, optical and electrical transport studies," Journal of Materials Science: Materials in Electronics, vol. 24, no. 9, pp. 3526-3535, 2013.

[23] A. Roy, A. Parveen, R. Deshpande, R. Bhat, and A. Koppalkar, "Microscopic and dielectric studies of $\mathrm{ZnO}$ nanoparticles 
loaded in ortho-chloropolyaniline nanocomposites," Journal of Nanoparticle Research, vol. 15, no. 1, article 1337, 2013.

[24] N. Gospodinova and L. Terlemezyan, "Conducting polymers prepared by oxidative polymerization: polyaniline," Progress in Polymer Science, vol. 23, no. 8, pp. 1443-1484, 1998.

[25] J. Tang, X. Jing, B. Wang, and F. Wang, "Infrared spectra of soluble polyaniline," Synthetic Metals, vol. 24, pp. 231-238, 1998.

[26] Y. H. Kim, C. Foster, J. Chiang, and A. J. Heeger, "Photoinduced localized charged excitations in polyaniline," Synthetic Metals, vol. 26, no. 1, pp. 49-59, 1988.

[27] M. G. Roe, J. M. Ginder, P. E. Wigeon, A. J. Epstein, M. A. Poulous, and A. G. Macdiarmid, "Photoexcitation of polarons and molecular excitons in emeraldine base," Physical Review Letters, vol. 60, p. 2789, 1988.

[28] A. Gruger, A. Novak, A. Régis, and P. Colomban, "Infrared and Raman study of polyaniline Part II: influence of ortho substituents on hydrogen bonding and UV/Vis-near-IR electron charge transfer," Journal of Molecular Structure, vol. 328, pp. 153$167,1994$.

[29] O. Misoon and K. Seok, "Effect of dodecyl benzene sulfonic acid on the preparation of polyaniline/activated carbon composites by in situ emulsion polymerization," Electrochimica Acta, vol. 59, pp. 196-201, 2012.

[30] C. Bian, Y. Yu, and G. Xue, "Synthesis of conducting polyaniline $/ \mathrm{TiO}_{2}$ composite nanofibres by one-step in situ polymerization method," Journal of Applied Polymer Science, vol. 104, pp. 21-26, 2007.

[31] J. Li, L. Zhu, Y. Wu, Y. Harima, A. Zhang, and H. Tang, "Hybrid composites of conductive polyaniline and nanocrystalline titanium oxide prepared via self-assembling and graft polymerization," Polymer, vol. 47, no. 21, pp. 7361-7367, 2006. 

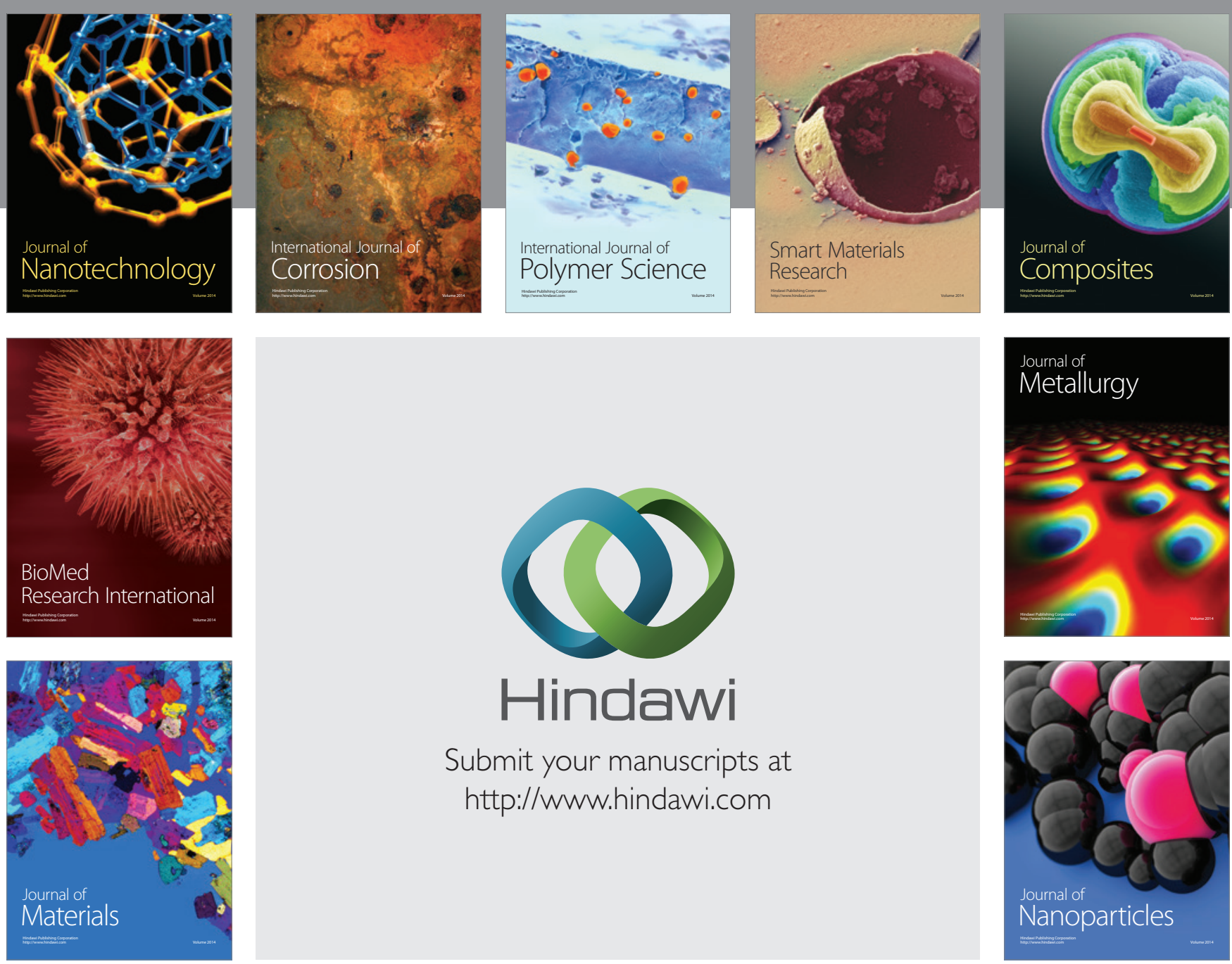

Submit your manuscripts at http://www.hindawi.com
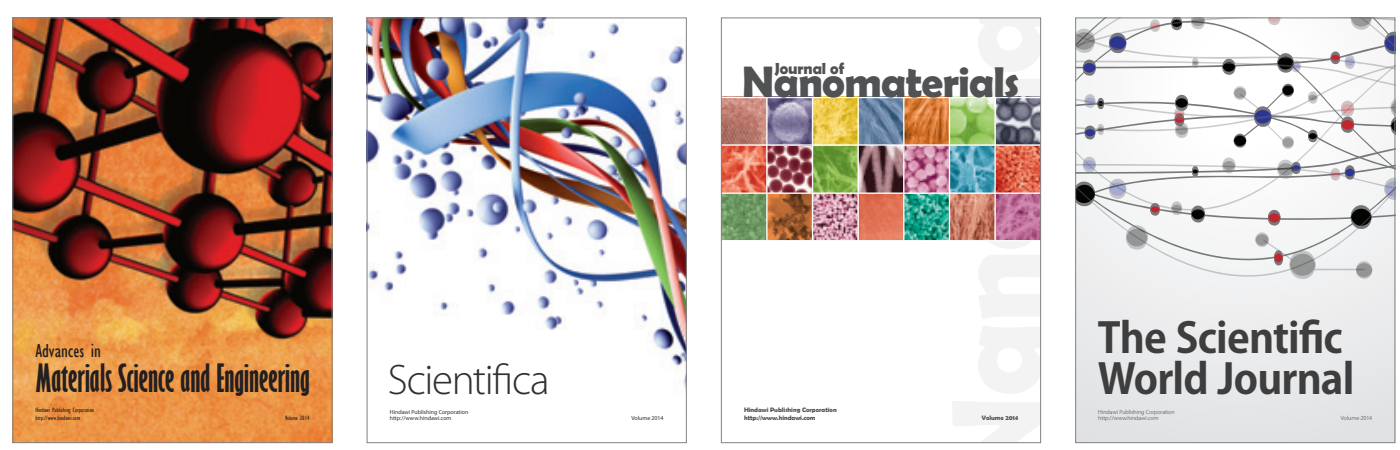

\section{The Scientific World Journal}
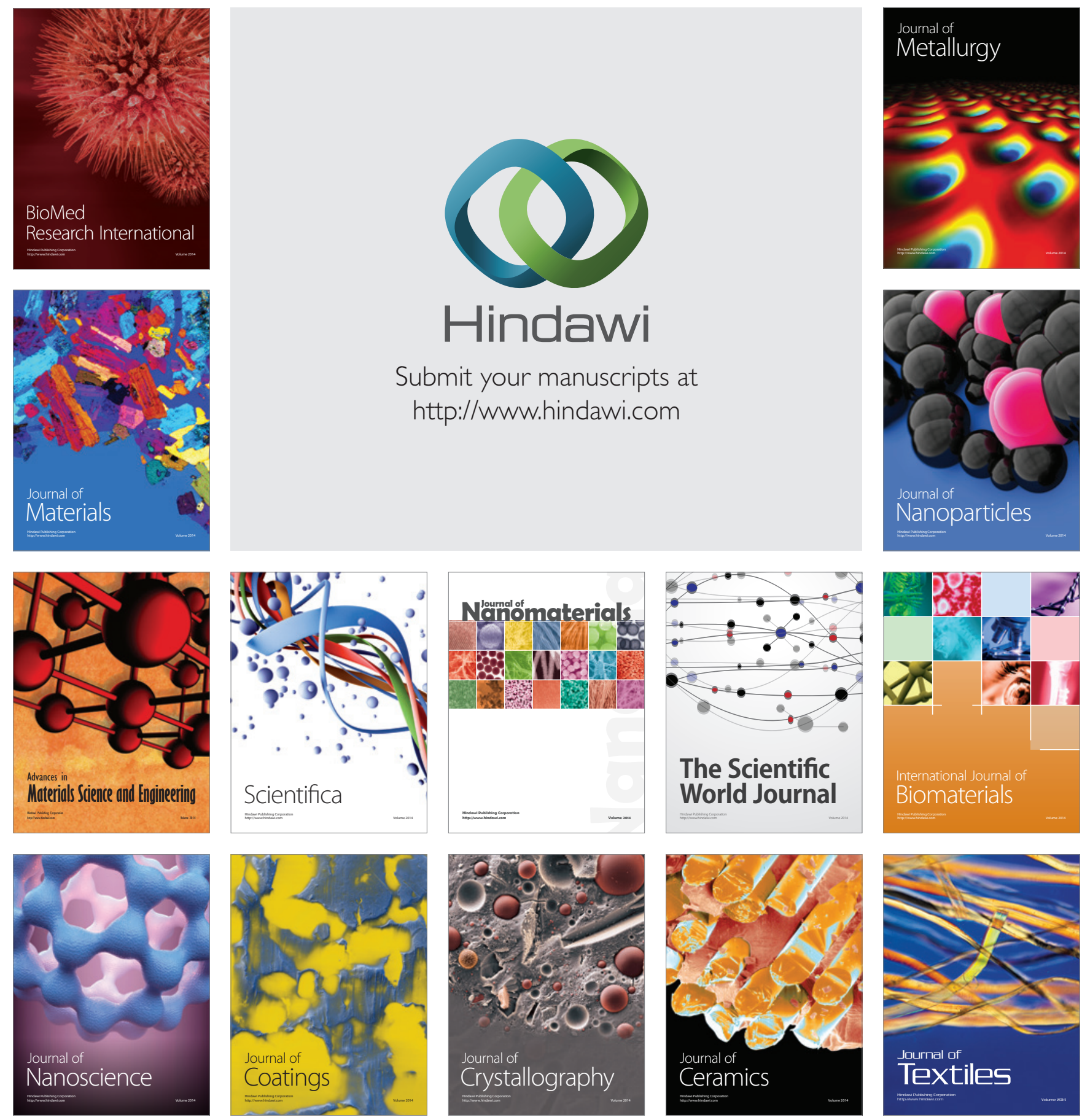